\title{
En la encrucijada del "sendero del medio": la dinámica de emplazamientos y desplazamientos discursivos en torno a la concepción del cuerpo en revistas de medicinas complementarias de la ciudad de Rosario*
}

\author{
Pablo Colacrai \\ Guillermo Ferragutti \\ Mauricio Manchado**
}

\section{Resumen}

El presente artículo se enmarca en una serie de avances producidos en un proyecto de investigación colectivo en el que, desde una perspectiva foucaultiana, realizamos un rastreo de las modalidades de ejercicio del poder y la resignificación de ciertas prácticas científicas, religiosas, políticas, médicas y pedagógicas en los discursos producidos desde las publicaciones alternativas a las terapéuticas tradicionales. En

${ }^{*}$ Artículo recibido 1 de abril de 2013. Aceptado 7 de octubre de 2013.

El presente trabajo es un avance de los resultados - parciales - obtenidos en el Proyecto de Investigación y Desarrollo de la Secretaría de Ciencia y Técnica de la UNR: "Cuerpo y bienestar: entre la promesa y el imperativo. Los discursos sobre el cuerpo en publicaciones que se presentan como alternativas a las terapéuticas tradicionales", dirigido por el Dr. Carlos Kuri. Departamento de Ciencias de la Comunicación, Facultad de Ciencia Política y RRII, Universidad Nacional de Rosario. Períodos 01/01/2010 al 31/12/2011 y 01/01/2012 al 31/12/2013.

** Pablo Colacrai es Licenciado en Comunicación Social por la Universidad Nacional de Rosario - Becario CONICET, Argentina. Doctorando Comunicación Social (UNLP). Contacto: pcolacrai@hotmail.com

Guillermo Ferragutti es Licenciado en Ciencias de la Comunicación por la Universidad Nacional de Rosario y Doctorando en Educación (UNLP). Argentina. Contacto: guillermoferragutti@yahoo.com.ar

Mauricio Manchado es Prof. Y Doctor en Comunicación Social Universidad Nacional de Rosario / CONICET (Argentina). Contacto: mauriom@steel.com 
este trabajo indagaremos, puntualmente, la dinámica de emplazamientos y desplazamientos que los discursos de dos publicaciones gráficas de medicina complementaria de la ciudad de Rosario ("El sendero del Medio" y "Do") establecen sobre el discurso médico científico. Discurso que en su superficie propone conciliar dos concepciones del cuerpo, $a$ priori, absolutamente disímiles: por un lado, el cuerpo como objeto separable del hombre y por tanto analizable y, por otro, el cuerpo como parte sustancial del hombre donde su materialidad parece ser borrada (visión holística, oriental y premoderna).

\section{Palabras clave}

Discurso médico/científico - cuerpo - medicina complementaria.

\section{Abstract}

This article is framed on a series of advances produced in a collective research project in which, from a foucauldian perspective, we tracked on the different modalities of exercising power and the resignification of scientific, religious, political, medical and pedagogical discourses produced in graphic publications of alternative therapy. In this paper, we inquire into what we can call the emplacement-displacement dynamics that these discourses in two alternative therapy publications in Rosario, Argentina -"The Middle Trail" and "Do"- established with medical and scientific discourses. Discourse this, in whose surface appears two conceptions of the body, which at first glance seem absolutely different. On the one hand, the body as a separable and therefore analyzable object owned by the man; and on the second hand, the body as a substantial part of men, in which there seems to be a completely erased materiality (holistic, eastern and pre-modern vision).

\section{Keywords}

Medical discourse/science - body - complementary medicine.

\section{Introducción}

En 1919 Kafka escribe "Un médico de campo", texto breve en el que cuenta los avatares de un médico que recorre diez millas para atender a un enfermo. Al llegar a la 
casa del paciente, más desanimado que entusiasmado, se dispone a revisarlo. Toca su cuerpo apoyando la cabeza sobre él y concluye que está sano: "Sólo una pequeña dificultad en el sistema circulatorio. Saturado de café por su madre, pero sano, y lo mejor sería sacarlo de un tirón de la cama. Como no me siento llamado a reformar el mundo lo dejo que siga acostado" (Kafka, 2005: 40). El médico cree haber terminado su tarea y se dispone a retirarse cuando la familia del enfermo, repentinamente, lo obliga a revisarlo otra vez. Ahora, y tras la nueva observación, descubre una herida de sangre y gusanos difícil de subsanar. "¿Me salvarás?", le pregunta el joven entre asustado y esperanzado, y la respuesta del médico — pensada, no emitida - será, particularmente, disparadora de este trabajo: "Siempre exigen del médico lo imposible. Han perdido su antigua fe. El párroco se queda en casa y destroza, una tras otra, sus casullas, pero el médico tiene que arreglarlo todo con su ligera mano de cirujano" (Kafka, 2005: 41).

El interrogante que inevitablemente surge es ¿qué tiene para decirnos esta afirmación? Pues, no sería tanto lo que dice sino lo que expresa, y lo que expresa es el imaginario de un orden social en el cual la ciencia ha pasado a ocupar un lugar primordial en la intervención del cuerpo. Pero, además, cristaliza un cambio de época o, en todo caso, una reafirmación de época, en la que el tándem cuerpo-ciencia se destaca por consolidar un campo de intervención para la medicina moderna, pero también por la importancia que ya ocupaba el cuerpo en el siglo XIX, en tanto potencia productiva y utilitaria. Para un orden moderno, que se ufanaba de mostrar grandes fábricas repletas de hombres en cadena, cárceles de multiplicidades ordenadas o formaciones militares derrochando orden y rectitud, el cuerpo era el centro y estaba en el centro de las discusiones, fundamentalmente, de un proyecto moderno que, por ejemplo en Argentina, necesitaba de los cuerpos para poblar $y$, en consecuencia, gobernar.

Ahora bien, ¿qué ha sucedido con esa sólida modernidad en la que el dispositivo médico-científico predominaba casi en soledad como discurso legitimador para hablar -e intervenir- sobre el cuerpo? Si hoy nos situamos en sociedades que, como ya lo hemos analizado en otro trabajo (Colacrai, Ferragutti \& Manchado, 2010) se caracterizan por su liquidez (Bauman, 2006), riesgo (Beck, 2000) e incertidumbre (Virno, 2008), nos interesa preguntarnos cuáles son los nuevos ordenamientos discursivos del tándem ciencia-cuerpo. $O$, en otros términos, ¿en qué materialidades discursivas podemos rastrear aquellas transformaciones?

Pues bien, una de nuestras hipótesis es que las revistas de medicina complementaria publicadas en la ciudad de Rosario (ver infra) expresan la emergencia y reactualización de discusiones cuyos antecedentes se remontan al siglo XVIII. Ya en 1741, la ortopedia de Andry planteaba la existencia de dos visiones sobre el cuerpo y, consecuentemente, dos formas - complementarias- de alcanzar su sanación o conservación. Una de ellas concebía al cuerpo como un objeto susceptible de ser corregido por el masaje modificador - corrección inducida - en el que, "a pesar de algunas denegaciones, la 
mano del pedagogo parece conservar con respecto al cuerpo del niño un sorprendente poder modelador" (Vigarello, 2005: 25), mientras que la otra visión estaba ligada a la idea de un cuerpo más "autónomo" que posee en su interior la fuerza oscura -y correctora- de la naturaleza; fuerza que será solo "orientada" por el terapeuta al detectar en las desviaciones corporales "analogías precientíficas" cuya eficacia terapéutica se da "mediante oscuras simpatías de sustancias." ${ }^{1}$ (Vigarello, 2005: 25).

Por lo tanto, el discurso de la primera ortopedia de Andry

todavía pertenece a dos 'mundos', que el futuro hará menos conciliables. Está repartido entre la visión de un cuerpo sometido al masaje modificador y la que lo abre a una mayor 'autonomía'. Los dos principios coexisten aquí sin que la contradicción sea claramente denunciada. Andry no puede abandonar las prácticas que un futuro próximo condenará o acallará, cuando su caducidad o su ineficacia sean admitidas. (Vigarello, 2005: 25)

Y, como decía el escritor norteamericano Mark Twain, "la historia no se repite pero rima", y en esa rima aparecen las mismas notas con diferentes melodías. Lo que aquí nos proponemos es repensar esa aparente contradicción que relucía ya en el siglo XVIII con la ortopedia de Andry donde el cuerpo asumía un doble carácter o condición². Pero ahora, en un nuevo orden social en el que la información $-\mathrm{y}$ allí el importante papel de los medios de comunicación - adquiere un lugar y una concepción diferente, $y$ en el que todas las certidumbres del mundo moderno son puestas en duda hasta tal punto que el dispositivo científico, en un mismo movimiento, es retomado y cuestionado en el interior de una lógica discursiva (la de las publicaciones que conforman nuestro corpus) que hace convivir concepciones del cuerpo a priori contradictorias.

Por todo esto, rastrearemos tanto las representaciones de cuerpo en las revistas de medicina complementaria ${ }^{3}$ de la ciudad de Rosario ("El Sendero de Medio" y "Do"), como las nuevas configuraciones que en ellas asume el discurso científico. En otro

\footnotetext{
${ }^{1}$ Por ejemplo, dirá Vigarello retomando a Andry: "el contacto de la espalda del niño con un pan que acaba de adquirir consistencia y rigidez podría ser portador de alguna virtud correctiva: 'Cuando el pan se saque del horno, quítesele de inmediato la cáscara que lo recubre y sobre ese pan caliente, que de todos modos se vigilará que no lo esté en demasía, tienda a su niño desnudo y de espaldas...". (Vigarello, 2005: 25)

${ }^{2}$ No proponemos rastrear en las revistas de medicina complementaria que componen nuestro corpus las visiones de cuerpo planteadas por Andry, sino la dinámica de coexistencia de dos principios -en apariencia - contradictorios que este autor, ya en el siglo XVIII, hacía convivir, sin conflicto alguno, bajo una misma lógica terapéutica.

${ }^{3}$ Hablaremos de "medicina complementaria" por ser la definición que los directores de la revista asignan al conjunto de prácticas publicadas. Más allá de las múltiples justificaciones sobre la utilización del término, existe una de orden jurídico que trasvasa a todas. Hablar de "medicinas alternativas" en lugar de "complementarias" implicaría postular a tales prácticas como "alternativas" - y por tanto sustitutivas—a la medicina tradicional. Esto conllevaría riesgos legales que son evitados con la nominación de "complementarias".
} 
trabajo (Colacrai, Ferragutti \& Manchado, 2011) hemos demostrado que la lógica sobre la que se sustenta el arsenal discursivo de estas revistas puede asimilarse a lo que Barthes (2005) llamó el discurso mitológico. Es decir, una suerte de segundo discurso que tiene como principal consecuencia la naturalización de una serie de valores. En ese momento detectamos cuatro matrices discursivas sobre las que se producía un continuo juego de emplazamientos y desplazamientos: el discurso político-liberal, el discurso místico-religioso, el discurso psicoanalítico y el discurso médico. En este artículo proponemos profundizar en la noción de cuerpo expresada en las revistas en tanto dicha expresión se presenta como efecto de la "crisis" de la medicina tradicional. Una "crisis" que no implica su desaparición, sino más bien desplazamientos inscriptos en nuevas formaciones discursivas como, por ejemplo, la de las medicinas complementarias.

\section{Breve descripción del corpus y algunas consideraciones de método}

La revista "Do" comenzó a editarse en septiembre de 2004 en forma bimestral, como una pequeña iniciativa familiar dedicada a la difusión de las artes marciales orientales. A partir del número 31, comenzó a dar un espacio a las "disciplinas complementarias", hasta comenzar gradualmente a ocuparse por entero de estas.

Sin embargo, conservó su nombre, una adaptación del sinograma Dō que se utiliza tanto en japonés como en coreano - cuyo equivalente chino es tao- y que, en Occidente, se ha popularizado como el sufijo que designa a las artes marciales. De hecho, yendo aún más allá, el término en los tres idiomas tiene una curiosa carga semántica: se trata tanto de un método como de una ética y estética específicas y rigurosas. Una actitud ante la vida y sus vicisitudes, abordadas mediante un grupo de saberes y de tradiciones; es frecuentemente traducido al castellano como camino.

Actualmente, el director de la revista conduce un programa televisivo llamado Nuevo Paradigma, que se emite por Canal 16, Televisión Regional (Cablevisión) los sábados de 20 a 21 y domingos 17 a 18, y un programa radial por Radio Fisherton CNN (FM 89.5 Mhz), de lunes a jueves de 23 a la una de la mañana.

Por su parte, la revista "El Sendero del Medio", de frecuencia mensual, lleva más de ochenta números editados en ocho años. Aunque al principio se presentaba como "periódico", la publicación adoptó el nombre de "revista" a partir del número 36 en marzo de 2008.

Durante estos años se le sucedieron innumerables cambios gráficos y de presentación, aunque la temática abordada nunca fue modificada sustancialmente. El equipo editorial también emite su programa en versión televisiva llamado "El Sendero del Medio TV", que se emite por canal 13 Somos Rosario (Cablevisión) los miércoles a las 18 y los domingos a las 20, y por radio, el "Sendero del Medio Radio", los martes de 20 a 22, por FM "Por Siempre" (FM 105.1 Mhz). Además, en 2011 la revista fue 
declarada de interés cultural por el Ministerio de Innovación y Cultura de la Provincia de Santa Fe por Resolución 331 del 27 de junio.

Ambas publicaciones sufrieron drásticas transformaciones debido a un notable crecimiento en muy pocos años de actividad. El Sendero del Medio, que inicialmente se imprimía a un color en papel obra, mejoró el gramaje del papel, incorporó la impresión a cuatro colores en papel fotográfico en tapa y contratapa, más una lámina intermedia, aumentó el número de páginas y la cantidad de avisos publicitarios. La revista Do, por su parte, se mantuvo algo más sobria, con una cantidad reducida de páginas, con un formato más pequeño, pensado para la distribución económica en dietéticas, gimnasios, sanatorios y clínicas privadas. Además de todo esto, como ya mencionamos, las dos iniciativas diversificaron su propuesta comunicacional hacia otros medios, como radio, TV, presencia en sitios Web, perfiles en redes sociales y canales en Youtube.

Respecto de los contenidos, El Sendero del Medio se publica en forma de monográficos, con temáticas abiertas tales como "la amistad", "el amor" o "el encuentro con los hijos", y todos los autores invitados escriben refiriéndose al tema. La revista Do, por su parte, trabaja simplemente sobre una nota central. Cabe mencionar, además, que los artículos que se escriben en ambas revistas tienen a sus propios anunciantes como autores, lo cual parece ser una búsqueda de un refuerzo intencional, ya que se colocan ambas piezas en la misma página.

Es importante mencionar también que en el proceso de recolección de datos nos encontramos con una diferencia significativa en lo que refiere a los espacios de circulación de ambas revistas, porque mientras "El Sendero del Medio" contaba con una distribución que iba desde las dietéticas hasta kioscos de revistas y librerías de la ciudad de Rosario, la revista "Do" definía un circuito de circulación mucho más reducido. Ante ello, una de las decisiones metodológicas adoptadas fue la de darle preponderancia en el análisis a la publicación "El Sendero del Medio"; importancia otorgada por la razón antes descripta pero también por el continuo crecimiento editorial de la revista reflejado no solo en el aumento de sus páginas sino también en las modificaciones introducidas al diseño y la producción periodística.

En ese sentido, el corpus seleccionado para la investigación consta de treinta números entre ambas revistas, publicados en el período 2005-2011, mientras que para la elaboración de este artículo (que presenta aquí una serie de avances o conclusiones parciales de dicha investigación) se seleccionaron los siguientes: El Sendero del Medio N. ${ }^{\circ} 21,22,23,24,33,34,35,36,38,43,44,45,52,53$ y Revista DO N. ${ }^{\circ} 34,37$ y 38.

En lo que refiere a los aspectos metodológicos, vale señalar que trabajamos desde un enfoque cualitativo y que la técnica de recolección de datos utilizada fue el "análisis de documentos". En un mismo sentido, para el análisis de los datos empíricos se llevó a cabo un proceso de codificación estándar a partir de etiquetas o membretes que nos permitieron identificar las marcas en las que emergían los juegos de emplazamientodesplazamiento discursivo trabajados en el presente artículo. Proceso, asimismo, 
realizado desde la perspectiva del análisis del discurso foucaultiano en el que concebimos a los discursos como "armas, como instrumentos de ataque y de defensa frente a unas relaciones de poder y de saber" (Foucault, 2006: 18) donde, en tanto que elementos o bloques de tácticas en la trama de las relaciones de fuerza, "puede haberlos diferentes e incluso contradictorios en el interior de la misma estrategia" y donde "pueden por el contrario circular sin cambiar de forma entre estrategias opuestas" (Foucault, 2008: 98).

En un primer momento, sin embargo, la estrategia de análisis de documentos parecía inadecuada para el estudio de estas revistas, ya que debido a la notoria convivencia de diferentes prácticas terapéuticas, las publicaciones no parecían tener un abordaje editorial concreto. ¿Qué piso epistemológico en común podían tener las Terapias de Mirar al Sol con el Brain Gym? ¿O la Moxibustión con las técnicas de Fotolectura? Así, se eligieron seis revistas al azar y se estableció un listado sin repeticiones de los distintos saberes y prácticas terapéuticas que aparecían. Al terminar, el listado incluía más de 280 entradas. Este breve pero significativo primer abordaje cuantitativo permitió despejar ciertas dudas respecto de lo que teníamos delante de nosotros. Se trataba de una publicación en la cual el eclecticismo era la regla.

Por último, una breve pero, creemos, necesaria aclaración de lectura. Entendemos el valor táctico de todos los discursos proferidos en sociedad, y consideramos que seguramente los enunciados presentes en las revistas bajo análisis se articulan sobre otros en diferentes espacios. No obstante, debemos anticipar que ninguna de las hipótesis y conclusiones manifestadas buscan sostenerse por fuera del análisis concreto de los textos que configuran nuestro corpus. Las conclusiones a las que conduce el trabajo no deben ser leídas en términos de verificación ni de posicionamiento respecto de las terapias complementarias, ni debe confundirse lo que dicen las revistas bajo estudio con lo que efectivamente sucede luego en este tipo de prácticas.

Nuestro análisis discursivo busca explicar de qué manera, y mediante qué articulaciones con otros enunciados, unas revistas de medicinas complementarias han llegado a ser un éxito editorial local, por lo que las conclusiones obtenidas hasta el momento son provisionales y solo basadas en los contenidos de la revista. En este sentido, una segunda etapa en la investigación buscará centrarse en los lectores y, mediante entrevistas semiestructuradas, contrastar nuestras hipótesis previas con lo relevado de ellos.

\section{Cuerpo y medicina}

Si miramos hacia el interior de la ciencia médica, veremos que el cuerpo nunca mantuvo una identidad conceptual clara y delimitada. Junto con la emergencia de los dispositivos disciplinarios, en el curso de la edad clásica, se configuró un modelo de intervención médica basado en la concepción del cuerpo como máquina. Las imágenes del cuerpo empiezan a construirse sobre un conjunto analógico de engranajes, 
mecanismos y articulaciones. La separación entre los contenidos del cuerpo y del alma constituye un registro anátomo-metafísico que Descartes ya se había ocupado de esbozar. A cada elemento de esta dualidad cuerpo-mente le corresponde su propio arsenal patológico, a partir del cual se elaboraron las enfermedades corporales y las enfermedades del espíritu. Pero subsiste, a la vez, un registro técnico-político constituido por un conjunto de procedimientos, reglas y técnicas destinado a controlar o corregir las operaciones individuales del cuerpo, y sobre el que se edificaron las instituciones estatales fundamentales de la sociedad moderna. A este conjunto de técnicas sobre el cuerpo le corresponde el nombre de disciplinas, entendidas como ese variado arsenal de métodos "que permiten el control minucioso de las operaciones del cuerpo, que garantizan la sujeción constante de sus fuerzas y les imponen una relación de docilidad-utilidad" (Foucault, 2002: 141), y que se han convertido, en los siglos XVII y XVIII, en unas formas generales y extendidas de dominación.

Sobre este registro técnico-político se constituyeron los procedimientos institucionales disciplinarios. Sobre el otro registro, el anátomo-metafísico, fueron definidos los límites y alcances de la medicina tradicional occidental. El modelo corporal del Hombre-Máquina de La Mettrie opera sobre estos dos niveles, tanto en términos de una reducción materialista del alma como de una teoría general de la educación (Foucault, 2002). La ciencia clásica exploró con mucho interés los alcances y las posibilidades reales del modelo corporal del Hombre-máquina, a través de la creación de autómatas que se constituyen no solo como modelos de funcionamiento del organismo, sino también como emergentes políticos que delimitan un esquema de preocupaciones y de ambiciones respecto del cuerpo.

El modelo corporal de Hombre-máquina que tomó la medicina clásica como arquetipo se construyó en diálogo permanente con todos aquellos aspectos del cuerpo que quedaban afuera de esta modelización. El Hombre-máquina ocupa la parte anatómica del dualismo anátomo-metafísico. Mientras tanto, la ciencia, durante mucho tiempo, desatenderá aquellos otros elementos irreductibles al modelo, más opacos a la experiencia, y que son justamente aquellos elementos metafísicos.

En términos políticos, el modelo de cuerpo-máquina tuvo un enorme grado de inserción en las sociedades industriales, que desarrollaron toda una serie de dispositivos, procedimientos y técnicas orientada específicamente a modelar pacientemente a sus ciudadanos a través de las variadas instituciones de encierro que componían el tejido social de los Estados nacionales, como las escuelas, los hospitales, las fábricas, las prisiones, los cuarteles y los asilos.

Estos procesos tuvieron como finalidad automatizar los procedimientos del poder, internalizarlos hasta el punto de garantizar un incremento en la fuerza corporal y su utilidad económica, a la vez que buscarán disminuir y subyugar estas mismas fuerzas en términos políticos, del mismo modo que aquellas dóciles maquinarias que reproducían a la perfección una y otra vez las acciones para la que estaban programadas y que 
obsesionaban a Federico II de Prusia. ¿Habrá descubierto alguna vez aquel engaño de Wolfgang von Kempelen, que el maravilloso Turco escondía bajo escena?

Sobre la base de este modelo, la medicina construyó la división funcional de los diferentes sistemas que involucraban al metabolismo, la respiración, el pensamiento y las sensaciones, la digestión, la circulación de la sangre, entre otros. Cada uno de estos sistemas poseía unidades con propiedades y funciones específicas. Consecuentemente, se construyó un abordaje de la enfermedad que atendía una sintomatología superficial y espontánea, la cual era señal de la afección de alguno de los elementos funcionales, e implicaba una cura posible a través de la restitución de la operatoria normal del cuerpo.

Para Sibilia (2005), retomando a Herminio Martins, este modelo de la medicina y la tecnociencia en general responde a lo que ella llama la tradición prometeica, cimentada en una firme confianza en el progreso, con el acento puesto en "la ciencia como 'conocimiento puro'", de la mano con "una visión meramente instrumental de la técnica" (44-45). Se trata de una tradición con firmes convicciones iluministas y positivistas, ya que predomina la fe en el progreso material y el perfeccionamiento constante de la técnica como elementos fundamentales de una sociedad racional, basada en el desarrollo científico-industrial. Este progreso no solo es lento y trabajoso, sino que está repleto de trampas, límites, caminos sin retorno, y debe ser recorrido con cuidado y precaución, como demuestra el mito de Prometeo, según el cual el conocimiento del fuego acercado por la mano del Titán les cuesta a los hombres la pérdida de la inmortalidad.

La famosa historia de Frankestein está manifiestamente inspirada en estas preocupaciones. El médico-creador atraviesa esos límites cuando conecta toscamente una serie de restos humanos, ensamblando una máquina humana a la que, a través de la electricidad (un descubrimiento que en ese entonces era reciente), devuelve al desmesurado monstruo la incomprensible llama de la vida, con consecuencias horrorosas. "La conclusión es evidente: los conocimientos y las técnicas de los hombres no son todopoderosos; sus 'dedos profanos' no pueden mancillar todos los ámbitos, porque hay límites que deben ser respetados" (Sibilia, 2005: 47). Ciertos asuntos pertenecen, entonces, a los dominios divinos y hay dimensiones de la vida que no serán objeto de la ciencia.

Estos límites, que la anatomía no puede explorar, quedan en la otra polaridad del registro: la metafísica, que construye sus campos de saber en permanente diálogo con ella. Del mismo modo, otra vertiente filosófica se mantuvo durante mucho tiempo a la zaga de la tradición prometeica: el proyecto fáustico.

Pero en cierto momento, a partir del siglo $\mathrm{XX}$, la clara frontera trazada entre la tradición prometeica y el proyecto fáustico comienza a desdibujarse, y la larga convivencia que habían mantenido entra en eclosión. Muchos de los límites autoimpuestos por la tradición prometeica empiezan a ser desbordados, la fe en la racionalidad humana y el progreso se agotan. El proyecto fáustico insiste en 
una dependencia, tanto conceptual como ontológica, de la ciencia con respecto a la técnica [...]. Los procedimientos científicos no tendrían como meta la verdad o el conocimiento de la naturaleza íntima de las cosas, sino una comprensión restringida de los fenómenos para ejercer la previsión y el control. (Sibilia, 2005: 50)

El impulso totalizante a la apropiación ilimitada marca una clara afinidad entre la ciencia fáustica y el espíritu del capitalismo. Insaciable e infinitista, el proyecto fáustico se revela como un impulso ciego de dominio y apropiación total de la naturaleza. Motorizado ya no por las prometeicas energías fósiles, sino por la energía sin límites contenida en el átomo, el proyecto fáustico amplía su dominio técnico sobre la naturaleza a partir de las tecnologías digitales y el carácter informacional de la electrónica, modelando el cuerpo de maneras totalmente metafísicas.

El cuerpo, modelado por el impulso metafísico del horizonte fáustico, es entendido como un obstáculo orgánico que restringe las potencialidades y ambiciones de los hombres, y anhela superarlas a través de la manipulación informacional. La presencia de un contenido discreto en forma de $A D N$, a la manera de un software informático presente en las células, implica un modelo ontológico del cuerpo totalmente nuevo, sobre el que la genética se ocupa, no sin una aspiración trascendental.

En este esquema de preocupaciones y modelaciones del cuerpo, esta nueva dimensión inmaterial, informacional, parece dejar atrás las antiguas limitaciones biológicas, y los nuevos avances técnicos buscan lograr un control total sobre la vida, tanto humana como no humana, donde los seres de la naturaleza se muestran como una materia manipulable, customizable.

La ciencia y la metafísica han logrado un punto de contacto en este tipo de poder sobre la vida, en el que los ingenieros de la biología se proponen reformular el mapa genómico de cada hombre, alterar el código genético y, así, ajustar la programación biotecnológica de cada individuo. Las enfermedades de este cuerpo ya no serían aquellas irritaciones a los órganos, a los cuales habría que restituir su funcionamiento normal, sino un peligroso enemigo microscópico, que puede alterar nuestro desconocido equilibrio genético y conducirnos a nuestra degeneración biológica. En ese sentido, resulta interesante el planteo de Nikolas Rose, quien sostiene que la novedad de la biopolítica contemporánea radica, a diferencia de la descripta por Foucault (1996) para los siglos XIX y XX,

en la percepción de que hemos experimentado una 'variación brusca', un aumento cualitativo de la capacidad de modificar nuestra vitalidad, nuestro desarrollo, nuestro metabolismo, nuestros órganos y nuestros cerebros [...] La vida humana se entiende ahora en el nivel molecular, es en ese nivel que es posible anatomizar los procesos vitales y alterar mediante procesos de ingeniería la vida. (Rose, 2012: 26) 
$Y$ es en ese nivel donde Rose inscribe lo que califica como una política de "la vida en sí y a una ciudadanía neuroquímica en la que se despliegan estrategias (por ejemplo, a partir de las nuevas tecnologías psiquiátricas y farmacéuticas) "para el gobierno del alma". Estrategias que obligan a los individuos "a involucrarse en una evaluación constante de riesgo, a supervisar y evaluar su humor, sus emociones y su cognición de acuerdo con un proceso más fino y más continuo de autoescrutinio" (Rose, 2012: 435). Sin la intención de realizar traslaciones mecánicas, creemos que los planteos de Rose habilitan una serie de claves conceptuales para pensar las estrategias discursivas puestas en juego en las revistas que analizamos.

\section{La duplicidad del cuerpo en las revistas}

Creemos que los discursos de la medicina complementaria que circulan en las revistas "El sendero del medio" y "Do" se pueden encuadrar dentro del esquema de debates de la medicina y la ciencia biológica de fines del siglo XX. Ya que en ellos, como veremos más adelante, con una lógica conciliatoria, persisten elementos tanto de un modelo corporal como del otro.

Como ya sostuvimos (Colacrai, Ferragutti \& Manchado, 2011), la idea misma de complementariedad es un síntoma de estas publicaciones, no solo una forma de llamarse a sí mismas. El desplazamiento de lo alternativo —que implicaría, necesariamente, una confrontación, una discusión- a lo complementario permite mantener un marco no polémico en contradicciones desplazadas que, vueltas a poner en su propio marco epistemológico original, significan profundas crisis y rupturas. Este es el fenómeno que se puede apreciar con relación a los diferentes cuerpos que construyen los textos de estas revistas.

El número 52 de la revista El sendero del medio está prácticamente en su totalidad dedicado a la problemática del cuerpo. Con el título "El encuentro con el Cuerpo Saludable" se subsume una multiplicidad de visiones que van desde las terapias quiroprácticas hasta las técnicas de concentración espiritual, pasando por lecturas adaptadas de la Biblia. Nos detendremos en algunos de estos textos para ejemplificar nuestra posición.

El artículo "Osteopatía: una alternativa sin medicamentos ni cirugía", escrito por el Lic. Roberto Meotto, describe en primer lugar el origen casi mítico de la técnica de curar dolores con las manos, para después afirmar:

la técnica evalúa al paciente en forma global comenzando por la postura y la marcha, para pasar luego a la evaluación analítica de los segmentos corporales que si se encuentran fuera de su mecánica normal, pueden generar cambios en el equilibrio orgánico que se traducirán a corto o largo plazo en dolor. (El sendero del medio $N{ }^{\circ}$ 52: 13) 
Y, más adelante:

se ha comparado a los practicantes de osteopatía como 'mecánicos' de la estructura corporal, y en efecto es así, ya que actúan devolviendo al cuerpo la capacidad de compensar o de devolver a su estado original cuando es agredido por un elemento externo (frío, caída, golpes, estrés), o interno (emoción).

Pero junto a esta visión física y mecánica del cuerpo, en la que son las malas posturas, la tensión o simplemente el frío o un golpe los que producen sus problemas, se encuentra un artículo sobre "Terapias avanzadas", en el que se afirma que "el cuerpo humano contiene los recursos para sanar y neutralizar las amenazas que podrían alterar su equilibrio" (El sendero del medio N. 52: 21). Por lo tanto, el mecanismo discursivo que -como regularidad - se nos presenta en dichas publicaciones es el siguiente: emplazamiento sobre una concepción de cuerpo prometeico -o modelo científico-si retomamos la clasificación propuesta por Sibilia (2005) y, al mismo tiempo, desplazamiento sobre dicha matriz discursiva para construir una concepción del cuerpo diferente (que identificamos en este artículo como modelo corporal del proyecto fáustico).

En el entrecruzamiento y convivencia de ambas concepciones, las terapias que calificamos como complementarias aseguran obtener asombrosos resultados en la curación de enfermedades psicosomáticas y en la recuperación de intervenciones quirúrgicas, ya que acceden directamente a "programas interiores que seguimos más allá de nuestra voluntad". Es decir, hay algo en nuestro cuerpo que se nos revela y que por medio de estas terapias puede revertirse. En el último párrafo el artículo expresa, sin ambages, su carácter de complementario: "Sin interrumpir los tratamientos médicos que corresponden a cada dolencia, podemos abordar rápida y eficazmente estas cargas emocionales, intelectuales y espirituales por medio de las Terapias avanzadas" (El sendero del medio N. ${ }^{\circ}$ 52: 21 ).

En cierto sentido esto no se presenta como una novedad si contemplamos que a mediados del siglo XX las neurociencias también complementaban ambas dimensiones, en apariencia contradictorias, y las hacían convivir "armónicamente". Precisamente Rose señala esto al describir que la psiquiatría biológica introduce una indistinción entre lo "orgánico" y lo "funcional" (o psicológico) cimentada en una "compleja base de conocimientos" (Rose, 2012: 373) y con el respaldo de un aparato de verdad ecléctico compuesto por diferentes especialidades y áreas de investigación.

Lo que el análisis de estos documentos (las revistas) nos permite identificar son una serie de marcas que, en tanto regularidades discursivas, definen y construyen múltiples visiones sobre el cuerpo; visiones que en las revistas conviven en la superficie misma de los enunciados tras una aparente armonía, a pesar de que sus postulados, de continuar su sucesión lógica, serían irreconciliables. 
$Y$ si inscribimos esta multiplicidad discursiva en las afirmaciones que sostienen quienes analizan las características de la biopolítica en la contemporaneidad, tanto a nivel internacional (Rose, 2012; Agamben, 2007) como local (Sibilia, 2005; Kaminsky, 2008; Papalini, 2006; 2007), observaremos que existe cierto consenso en sostener que lo que se produce es "una auto-vigilancia perpetua sobre el propio cuerpo, y una negociación consciente, individual y constante, entre los riesgos y placeres que involucra cada práctica corporal" (Sibilia, 2009: 195). Por lo tanto, la multiplicidad de visiones sobre el cuerpo tras las regularidades discursivas que identificamos son la expresión de esa tensión-negociación o contradicción-complementación que podemos identificar en los fragmentos citados, como también la cristalización de una práctica del self (o política de sí mismo) en la que opera el juego de emplazamientodesplazamiento sobre las matrices científicas sin que ello implique una tensión o contradicción paralizante.

En un mismo sentido, en un artículo acerca de los Registros Akáshicos por medio de los cuales se pueden detectar los órganos en los que se alojan las emociones que nos lastiman, se afirma que: "La medicina nos ayuda desde su ciencia, la psicología desde el campo mental, Registos Akáshicos nos guía y aporta desde el campo espiritual" (El sendero del medio N. ${ }^{\circ}$ 52: 31). Vemos una vez más la complementariedad emergiendo en la superficie de los discursos, aun cuando la relación entre los elementos sea difícil de explicar. El cuerpo es fraccionable, ya que se puede detectar exactamente el órgano dañado. Pero al mismo tiempo es un cuerpo medicalizado y medicalizable, no exento, por lo que vemos, de un inconsciente que deberá ser tratado por un psicoanalista. Entonces, ¿cómo es ese cuerpo que puede ser intervenido por medio de este tratamiento? "El secreto - termina el artículo- es estar conectados con nuestra esencia, desde allí no nos equivocamos, porque cuando vibramos con la energía del amor, que nos constituye como seres de luz que somos, conservamos la armonía y, por lo tanto, la salud." Cuerpo orgánico, máquina, cambiable, reemplazable, curable, vehículo de la existencia, compañero de ruta, pero al mismo tiempo, etéreo, inasible, repleto de energías, de luz, de vibraciones.

En esta dinámica de emplazamientos y desplazamientos, se abre todo un nuevo campo de consecuencias posibles sobre el cuerpo. Los límites de la desviación se van desdibujando y pequeñas actividades pueden tener graves resultados biopolíticos. Así, desde la nutrición depurativa, uno de los autores diagnostica en un artículo sobre calidad reproductiva que "en EE. UU. nace un bebé anormal cada 5 minutos" (EI sendero del medio, N. ${ }^{\circ}$ 53: 22). Luego, se sincera y exclama "iiiPor favor, basta de 'mal paridos'; el mundo ya está repleto de ellos!!!" (23). Apoyándose en una voz más autorizada e impersonal, el autor asegura que

la ciencia se está percatando de que la calidad del óvulo y del espermatozoide que crean una nueva vida, dependen, como no podía ser de otro modo, de la calidad y el 
orden de los organismos generadores. Qué podemos esperar de padres que fuman, se alcoholizan, toman drogas (farmacológicas y de las otras), consumen alimentos no fisiológicos, están expuestos a químicos tóxicos, consumen violencia y no realizan prácticas depurativas. (El sendero del medio, N. ${ }^{\circ}$ 53: 23)

Allí queda presentado, entonces, el mecanismo discursivo. Por una parte, se diagnostica un problema, de orden global y multifactorial, pero relacionándolo solo con un número limitado de causas. Luego, se establece un orden de consecuencias biopolíticas en el que la ingesta de alcohol supone un peligro para la descendencia, quedando para el final la propuesta de una práctica nutricional depurativa. Pero lo interesante de todo este procedimiento es que sin el anclaje de un modelo corporal elástico y adscripto simultáneamente a ambas concepciones, no es posible establecer este orden de consecuencias flexibles ni la preocupación por la descendencia mal nacida.

Para finalizar, un último ejemplo del mecanismo de emplazamiento-desplazamiento que procuramos describir en el transcurso del artículo. En la nota "Mandalas para el bienestar", la autora (Farm. Alicia Depiante) sostiene que "dibujar y pintar mandalas nos ayuda a hacer conscientes nuestras emociones, produciendo diversas respuestas neuroquímicas en nuestro cuerpo. Con la liberación de endorfinas se genera placer, relajación, bienestar y se posibilita atenuar el estrés, la ansiedad, la depresión" $Y$, más adelante: "el mandala nos abre las puertas hacia nuestro 'sanador interno', nos proporciona un refugio donde sentirnos a salvo del mundo exterior lleno de estrés y de confusión, y nos ayuda a encontrar paz y calma" (El sendero del medio N. ${ }^{0}$ 52: 35).

Acaso nos encontremos, en estas revistas, frente al paroxismo del encuentro entre universos discursivos que sumidos en el juego de los emplazamientosdesplazamientos, nos presentan un cuerpo etéreo que necesita su reafirmación física, o un cuerpo espiritual que necesita de la materialidad para despojarse de ella. Lo que esta dinámica de emplazamiento-desplazamiento habilita es el borramiento de la tensión (tras la lógica de la complementariedad) que puede existir entre el discurso científico y el no-científico, en la conjunción de dos modelos corporales significativamente distintos pero que conviven en un mismo plano discursivo. Por lo tanto, podemos afirmar que la disputa entre los dos modelos corporales descriptos en el transcurso de este trabajo no es novedosa, pero en la textualidad de las revistas de medicina complementaria se configuran, tras la articulación de emplazamientodesplazamiento, escenarios donde el conflicto y la tensión pasan desapercibidos.

Articulación inscripta además en la repetición de discursos existentes en la cultura; discursos que ya tienen, de alguna manera, su propio estatuto de verdad y a partir de los cuales se puede explicar el poder mágico de los mandalas por la "conocida" liberación de endorfinas, para después concluir que ese mismo procedimiento nos abrirá las puertas a nuestro sanador interno. Sanador interno y endorfinas puestos al 
mismo nivel del argumento, la supuesta veracidad de uno, afirmado por la maquinaria médico-científica, se desplaza al otro, amalgamando elementos que pertenecen, a universos discursivos $-\mathrm{y}$, por consiguiente, argumentales y epistemológicos- muy diferentes.

\section{A modo de conclusiones: de la complementariedad o de un mundo sin conflictos}

En términos metodológicos, los instrumentos y técnicas utilizadas funcionaron correctamente. Se revela como necesaria la entrada de la investigación en una segunda etapa, mediante el abordaje del punto de vista de los lectores, contrastar hipótesis, corregir ideas y establecer la distancia entre lo que las revistas dicen y lo que los lectores leen. ¿Persistirá esta idea de lo complementario en los lectores de la revista? ¿Qué criterios establecen ellos para elegir entre una y otra práctica terapéutica? ¿Qué visiones tienen frente a la medicina tradicional?

Hemos analizado en trabajos anteriores la invocación al peligro y el par miedoangustia que definen las revistas (Colacrai, Ferragutti \& Manchado, 2010), así como la apelación a la responsabilidad individual y la interioridad como refugio ante las inseguridades de lo que Beck (2000) llamaba sociedades de riesgo. Hemos también caracterizado la dinámica de los desplazamientos-emplazamientos discursivos sobre nociones ampliamente aceptadas por la sociedad, en cuatro ejes de análisis: el político, el místico-religioso, el psicológico y el médico (Colacrai, Ferragutti \& Manchado, 2011). Sobre este último eje nos interesó, particularmente, el abordaje del cuerpo como categoría del saber anatómico por excelencia, y allí vimos que los modelos contrapuestos que han generado las mayores "crisis" en la medicina tradicional coexisten de modo conciliador en los textos, bajo el paraguas de lo complementario.

Esta idea de complementariedad es lo que permite una coexistencia tal. Mientras los saberes mencionados se mantengan en su superficialidad lógica no debe temerse que haya riesgos de contradicción, porque algo los protege, y eso es la complementariedad. Se trata de una negación de todo conflicto, ninguna práctica cae en desuso ni ninguna es superada, ni por algún avance técnico ni científico. Algunas incluso obtienen parte de su legitimación en que se trata de terapias ancestrales, mientras otras utilizan los últimos hallazgos científicos para justificar la importancia de su práctica. Como en el Aleph de Borges, en estas revistas todo está junto, al mismo nivel y con el mismo grado de importancia. El antiguo debate entre los dos cuerpos, o entre las dos formas de construir al cuerpo del hombre, se desvanece en la secuencia de artículos que por momentos afirman que el cuerpo es algo externo, algo propio, algo que debemos cuidar, algo que nos fue dado o que nos pertenece o que debemos olvidarlo, algo que puede asemejarse a un vehículo, o un estuche que contiene nuestro verdadero yo, pero también algo que somos nosotros, algo en donde se pueden leer los signos de nuestros problemas o algo que, a la vez, nos los provoca. 
Parece, pues, que el descubrimiento que estas revistas intentan transmitir —no tanto en el enunciado de sus textos, sino en el nivel de la enunciación y, sobre todo, en la superposición de enunciados contradictorios-, es que no hay razones para elegir, no hay motivos para adoptar tal o cual posición, el cuerpo puede ser una máquina y al mismo tiempo un cúmulo de energías, podemos ser seres de luz y podemos, también, albergar esa luz en nuestro ADN. En suma, existe un nuevo camino en el que se suturan y superan todos los conflictos: el sendero del medio.

\section{BIBLIOGRAFÍA}

Barthes, R. (2005). Mitologías. Buenos Aires: Siglo XXI.

Bauman, Z. (2006). Modernidad líquida. Buenos Aires: Fondo de Cultura Económica.

Beck, U. (2000). Retorno a la teoría de la "sociedad del riesgo". Boletín de la AGE, 30. Disponible en: http://age.ieg.csic.es/boletin/30/01.pdf

Colacrai, P., Ferragutti, G. \& Manchado, M. (2010). Sálvese quien pueda: La Sociedad de la medicina complementaria. Memorias de las XIV Jornadas Nacionales de Investigadores en Comunicación. Disponible en: http://redcomunicacion.org/memorias/pdf/2010cocolacrai pablo.pdf

Colacrai, P., Ferragutti, G. \& Manchado, M. (2011). La intertextualidad 'oculta' de la medicina complementaria. Memorias de las XV Jornadas Nacionales de Investigadores en Comunicación. Disponible en: http://redcomunicacion.org/memorias/pdf/fecolacraiferraguttimanchado.pdf

Ferrer, C. (2004). La curva pornográfica. El sufrimiento sin sentido y la tecnología. Revista Artefacto, 5. Buenos Aires.

Foucault, M. (1996) Genealogía del racismo. La Plata: Altamira.

Foucault, M. (2008) Historia de la sexualidad: 1. La voluntad de saber. Buenos Aires: Siglo XXI.

Foucault, M. (2002). Vigilar y Castigar. Buenos Aires: Siglo XXI.

Foucault, M. (2006) Yo, Pierre Riviére, habiendo degollado a mi madre, a mi hermana y a mi hermano... Barcelona: Tusquets.

Giorgi, G. \& Rodriguez, F. comp. (2007) Ensayos sobre biopolítica: excesos de vida. Buenos Aires: Paidós.

Kafka, F. (2005). Un médico de campo. En F. Kafka: Relatos completos, 2. Buenos Aires: La Página/Losada.

Kaminsky, G. et ál. (2008) Bartleby: preferiría no. Lo bio-político, lo post-humano. Buenos Aires: La Cebra.

Papalini, V. (2006) Literatura de autoayuda: Una subjetividad del Si-Mismo enajenado, en La Trama de la Comunicación, Vol. 11, Rosario: UNR Editora.

Papalini, V. (2007) La domesticación de los cuerpos, en "Enlace: Revista Venezolana de información, tecnología y conocimiento", año 4, núm.1, Universidad de Zulia, Venezuela. 
Rose, N. (2012) Políticas de la vida: biomedicina, poder y subjetividad en el siglo XXI. La Plata: UNIPE: Editorial Universitaria.

Sibilia, P. (2005). El hombre postorgánico. Cuerpo, subjetividad y tecnologías digitales. Buenos Aires: Fondo de Cultura Económica.

Sibilia, P., Villagran, J. \& Olaechea, B. (2009) Sobre el cuerpo y la comunicación: Entrevista con Paula Sibilia en Revista Educación Física y Ciencia, año 11, La Plata.

Vigarello, G. (2005). Corregir el cuerpo. Buenos Aires: Nueva Visión.

Virno, P. (2008). Gramática de la multitud: para un análisis de las formas de vida contemporáneas. Buenos Aires: Colihue. 